\title{
Retinal Vessel Segmentation Based on Adaptive
}

\section{Random Sampling}

\author{
Nicolas Jouandeau, Zhi Yan and Patrick Greussay \\ Advanced Computing Laboratory of Saint-Denis (LIASD) \\ Paris 8 University \\ 93526 Saint-Denis, France \\ Email: \{n, yz,pg\}@ai.univ-paris8.fr \\ Beiji Zou and Yao Xiang \\ School of Information Science and Engineering \\ Central South University \\ Changsha 410083, PR China \\ Email: \{bjzou,yao.xiang\}@mail.csu.edu.cn
}

\begin{abstract}
This paper presents a method for the extraction of blood vessels from fundus images. The proposed method is an unsupervised learning method which can automatically segment retinal blood vessels based on an adaptive random sampling algorithm. This algorithm consists in taking an adequate number of random samples in fundus images, and all the samples are contracted to the position of the blood vessels, then the retinal vessels will be revealed. The basic algorithm framework is presented in this paper and several preliminary experiments validate the feasibility and effectiveness of the proposed method.
\end{abstract}

Index Terms-Blood vessel segmentation, unsupervised learning, sampling algorithm, retinal vessels, fundus images

\section{INTRODUCTION}

Retinal vessel segmentation refers to extract blood vessels in retinal camera images. This technique is of important significance on screening, diagnosing and treating various ophthalmological and cardiovascular diseases. Manual segmentation of retinal blood vessels can guarantee the accuracy of the segmentation, but it is a long work which also needs some professional trainings. The public desires to achieve automation by means of computers. This paper introduces a new method for automatic retinal vessel segmentation based on an adaptive random sampling algorithm. The basic idea is (see Figure 1): take a random sample (i.e. a random pixel) $p$ in the fundus image, if there exists a pixel $q$ around $p$ which has a lower value (i.e. the color of $q$ is darker than that of $p$ ), then $p$ retracts to $q$ and marked as vessel. This method is actually to find the local optimal solutions, then combined into a global optimal solution.

The rest of the paper is organized as follows: Section II describes an overview of some related works; Section III describes our adaptive random sampling algorithm; Section IV presents the experimental results obtained with the proposed method; and the paper is concluded in Section V at last.

\section{RELATED WORKS}

Retinal vessel segmentation is actually an issue of image processing, which has been widely studied since the late 1980s. Existing methods can be generally divided into the following categories [1]: 1) machine learning (mainly including supervised learning and unsupervised learning), 2) matched filtering, 3) vessel tracking, 4) morphological processing, 5) multi-scale approaches, 6) model based approaches, and 7)parallel hardware based approaches. Experiments show that the accuracy of segmentation can reach up to $97 \%$.

However, among the existing works, the development of unsupervised learning methods is relatively small, and the accuracy of segmentation by using these methods is relatively low. [2] presented an unsupervised method for segmenting the vessel from color retinal images. The vessels are modeled as trenches and the medial lines of the trenches are extracted using the curvature information derived from a novel curvature estimate. The output of this process is in the form of a medial axes map of the vessels. The entire vessel structure is then extracted using a region growing method. Their method achieves an area under the ROC (Receiver Operating Characteristic) curve of 0.9271 and a maximum average accuracy of 0.9361 on the publicly available DRIVE database. [3] presented another unsupervised method by extracting the intensity information from red and green channels of the same retinal image to correct non-uniform illumination in color fundus images. Matched filtering is utilized to enhance the contrast of blood vessels against the background. The enhanced blood vessels are then segmented by employing 
1: while a maximum number of pixels $n$ pixels has been marked as blood vessel do

2: $\quad$ for number of sample $\mathrm{i}=0$ to maximum number $\mathrm{m}$ do

3:

image

4:

threshold then

5:

6:

7:

8:

9:

10:

11: end for

12: end while

generate a random sample $\mathrm{p}$ in retinal if $\exists \mathrm{q} \in$ adjacent area $\alpha$ of $\mathrm{p}:|\mathrm{p}-\mathrm{q}|>$ if $\mathrm{q}<\mathrm{p}$ then $q$ is labeled as vessel else p is labeled as vessel end if end if spatially weighted fuzzy c-means clustering. The evaluation of the method resulted in an area under the ROC curve of 0.9518 and 0.9298 , and an average accuracy of 0.8911 and 0.8976 , respectively on the DRIVE and STARE databases.

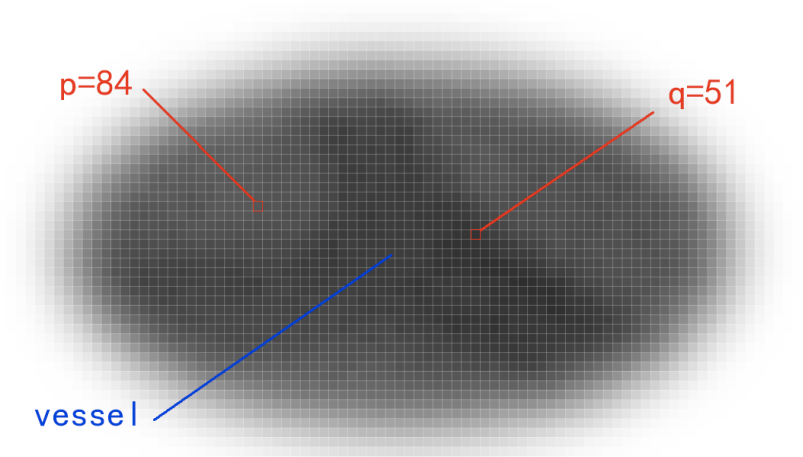

Fig. 1 An example illustrating the sampling-based method
This manner of manual setting must be difficult to deal with a large number of images. It will be changed to an automatic manner in the program according to various images in our future implementations.

\section{EXPERIMENTS}

Our proposed method is inspired by the sampling-based methods in the field of robotics [4]. Sampling-based methods are currently considered state of the art for robot motion planning in high-dimensional spaces, and have been highly successful in solving many complex problems which have dozens or even hundreds of dimensions. These methods can avoid the problem of local minima, but they are probabilistically complete, meaning they cannot guarantee to find a solution if one exists. The fundus image could be considered to be a complex environment. Using a probabilistic method can meet the requirement of high efficiency and low consumption.

Our method for retinal vessel segmentation by using the adaptive random sampling algorithm is illustrated in Algorithm 1.

In Algorithm 1, line 1 means that the program will bee repeated until convergence has been reached. Line 2 indicates that we must take a sufficient number (denoted by $m$ ) of sample pixels. In line $4,|p-q|$ indicates the difference between the two pixel values $\mathrm{p}$ and $\mathrm{q}$. Line 6 can actually be regarded as the retraction of the sample $p$ to the location of the pixel q. That is the reason why we call this algorithm the adaptive random sampling. In the current version of the algorithm, $\mathrm{n}, \mathrm{m}$ and threshold are three variables that have been assigned a value at the beginning of the program.

\section{A. Experimental Setup}

Several primary experiments have been conducted to evaluate our proposed method. All experiments reported in this paper were carried out on the Ubuntu 10.044 operating system with an Intel Core 2 Duo T5500 1.66GHz processor, an Intel 945GM Express chipset and two DDR2 $800 \mathrm{MHz} 512 \mathrm{MB}$ dual channel memory. Our program is realized with secondary development tools based on libpng library, which can be downloaded from www.ai.univ-paris8.fr/ $\sim$ yz/ARS.tar.gz.

\section{B. Experimental Results}

The first stage of the experiment is to transfer a color fundus image to a target image. This step is essential to vessel segmentation because it is meaningful to find an effective objective metric [5]. In our method, the color fundus image is converted to a magenta channel image in CMYK (Cyan, Magenta, Yellow and blacK) color model. In contrast with the green channel image in RGB (Red, Green and Blue) color model, greyscale image, and color image, retinal vessels are more distinct in the magenta channel image in CMYK color model [6]. The magenta channel image is also more suitable for our adaptive random sampling algorithm, by reason of the obvious difference between vessels and other areas, thus more sensitive to the threshold. A visual comparison of the different images is given in Figure 2. 


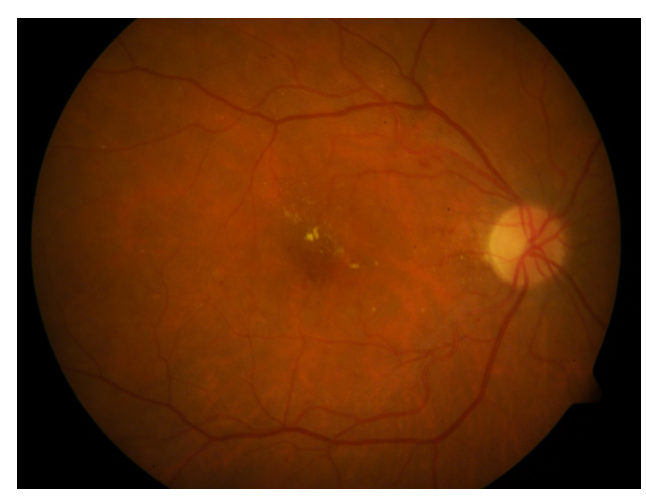

(a) Original color image

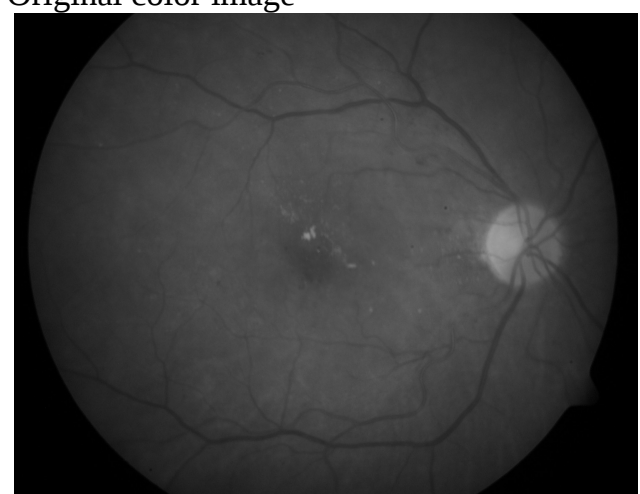

(b) Greyscale image

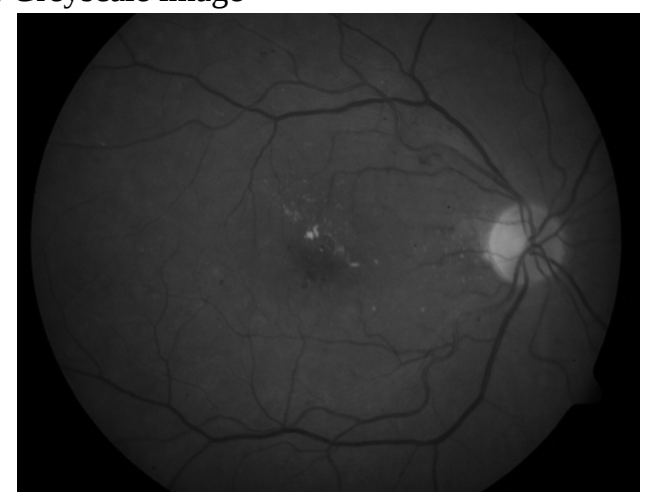

(c) Green channel image (RGB color model)

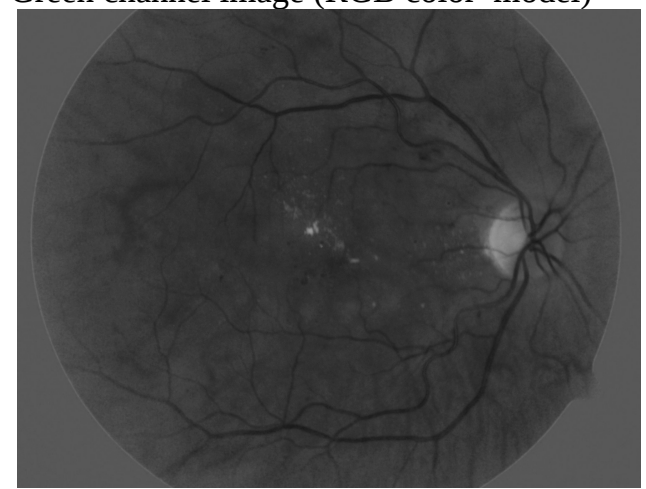

(d) Magenta channel image (CMYK model)

Fig 2 : A visual comparison of different models of a fundus image

The experimental fundus image is selected from the ImageRet database [7]. Figure 3 shows the experimental results for four critical areas in the image. These four areas are sequentially labeled as area (A), area (B), area
(C) and area (D), in accordance with the clockwise direction. Area (A) represents the vessel branch and cross, area (B) represents the blood vessels on optic disc, area (C) represents the central vessel reflex, and area (D) represents the blood capillaries. As can be seen from Figure 3, the current version of our method can identify vessel branch and cross, vessels on optic disc, and central vessel reflex effectively, but it cannot handle blood capillaries very well, because the pixel values of the capillary are too close to the values of the other area. This problem will be improved in our future research. Figure 4 depicts the vessel extracted by using our sampling-based method. The left image (subfigure (a)) represents the initial result. The vessels can be obviously identified from this image, but it still has a lot of noises for reasons of uneven background, macular and exudates. The right image (subfigure (b)) represents the result after preliminary noise reduction. In fact, the noises produced by using the current version of our method are mainly grouped around the macular and the exudates. From another point of view, it can be used in detection of pathology.

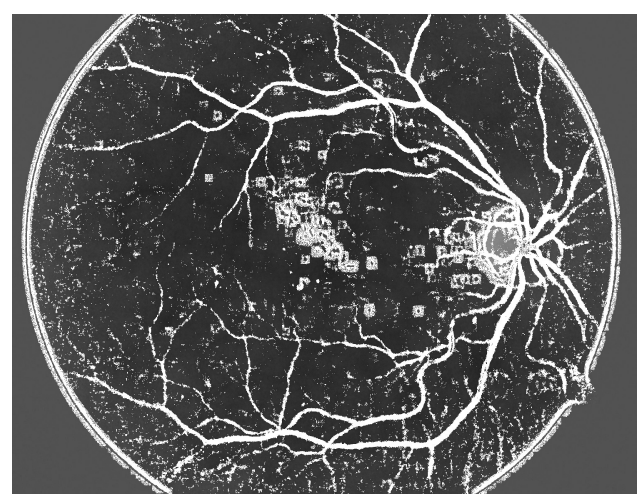

(a) Initial result

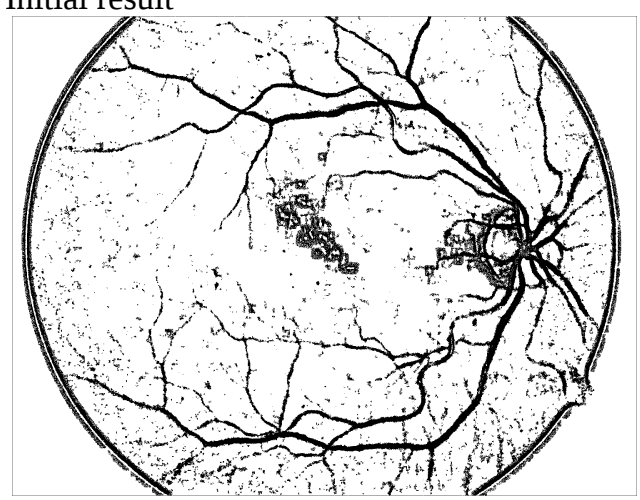

(b) After preliminary noise reduction

Fig 3 : Vessel extracted by using the sampling-based method 


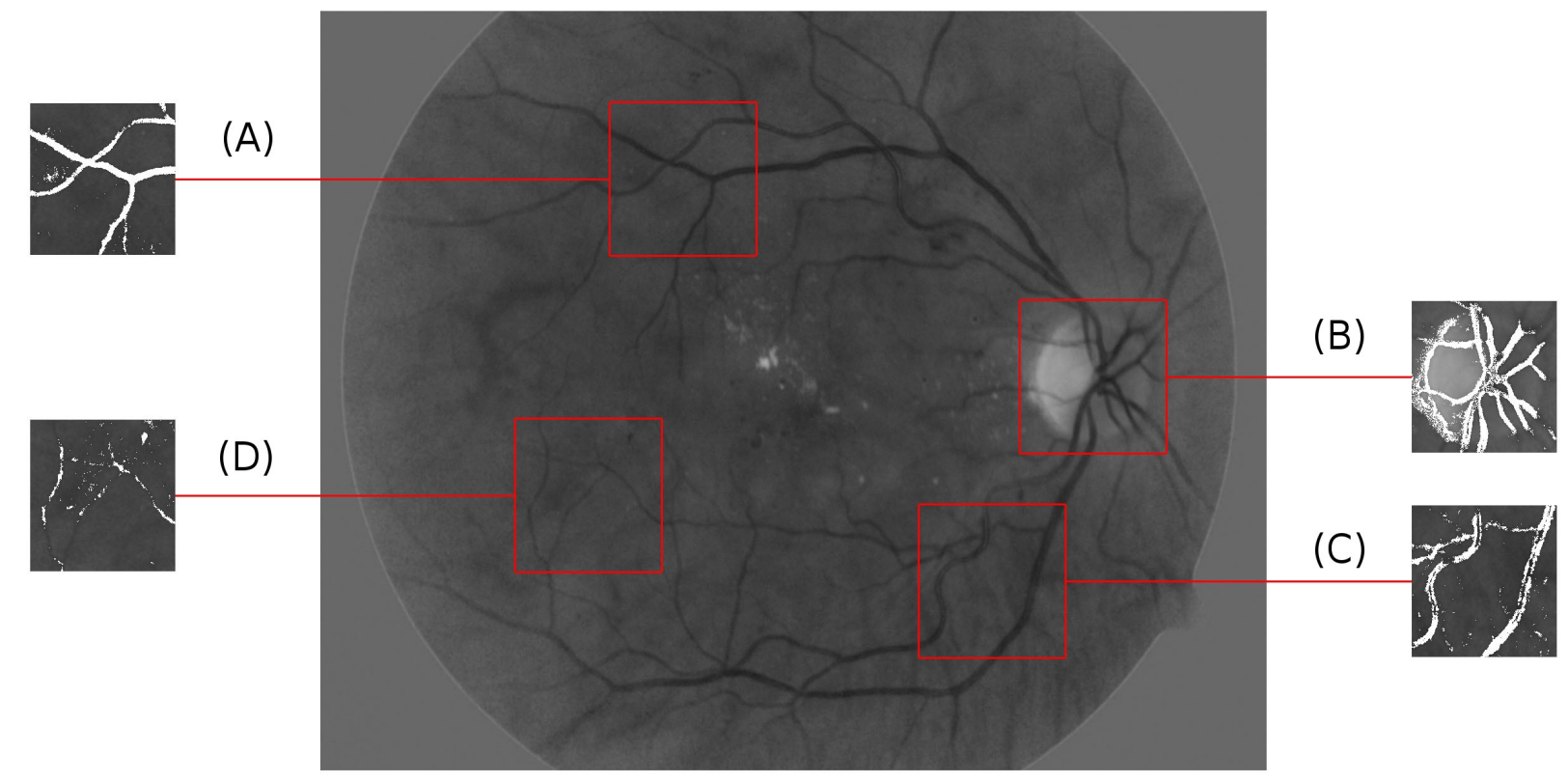

Fig4 : Four critical areas in a fundus image. Area (A) represents the vessel branch and cross, area (B) represents the optic disc, area (C) represents the central vessel reflex, and area (D) represents the blood capillary.

\section{CONCLUSION}

In this paper, we presented a new retinal vessel segmentation method based on an adaptive random sampling algorithm. The idea is to take an adequate number of random samples in retinal camera images, and all the samples should retract to the location of the vessel so as to present it. A basic algorithm framework and some preliminary experimental results are presented. The experimental results proved the feasibility of our proposed method. The future work will focus on:

- Algorithm need to be improved, especially the determination of the values of $\mathrm{n}, \mathrm{m}$ and threshold.

- Image noise should be reduced effectively.

- More images need to be tested, and experimental results should be quantified with accuracy.

\section{ACKNOLEDGMENTS}

This work has partly been supported by the Sino-French scientific project "Xu Guangqi" under contract number PHC 28011WC.

\section{REFERENCES}

[1] M. Fraz, P. Remagnino, A. Hoppe, B. Uyyanonvara, A. Rudnicka, C. Owen, and S. Barman, "Blood vessel segmentation methodologies in retinal images - a survey," Computer Methods and Programs inn Biomedicine, vol. 108, no. 1, pp. 407-433, October 2012
[2] S. Garg, J. Sivaswamy, and S. Chandra, "Unsupervised curvature-based retinal vessel segmentation," in Proceedings of the 4th IEEE International Symposium on Biomedical Imaging (ISBI'07), Washington DC, USA, April 2007, pp. 344-347.

[3] G. B. Kande, P. V. Subbaiah, and T. S. Savithri, "Unsupervised fuzzy based vessel segmentation in pathological digital fundus images,” Journal of Medical Systems, vol. 34, no. 5, pp. 849-858, October 2010.

[4] Z. Yan, N. Jouandeau, and A. Ali Cherif, "ACS-PRM: Adaptive cross sampling based probabilistic roadmap for multi-robot motion planning," in Proceedings of the 12th International Conference on Intelligent Autonomous Systems (IAS’12), Jeju Island, Korea, June 2012.

[5] Y. Xiang, B. Zou, and H. Li, "Selective color transfer with multi-source images," Pattern Recognition Letters, vol. 30, no. 7, pp. 682-689, 2009.

[6] W. Wang, J. A. Ozolek, and G. K. Rohde, "Detection and classification of thyroid follicular lesions based on nuclear structure from histopathology images," Cytometry A, no. 77A, pp. 485-494, 2010.

[7] T. Kauppi, V. Kalesnykiene, J.-K. Kamarainen, L. Lensu, I. Sorri, A. Raninen, R. Voutilainen, H. Uusitalo, H. Kalviainen, and J. Pietil a, "DIARETDB1 diabetic retinopathy database and evaluation protocol," in Proceedings of the 11th Conference on Medical Image Understanding and Analysis (MIUA 2007), Bristol, UK, July 2007, pp. 61-65. 